\title{
All Master Integrals for Three-Jet Production at Next-to-Next-to-Leading Order
}

\author{
D. Chicherin, ${ }^{1}$ T. Gehrmann, ${ }^{2}$ J. M. Henn, ${ }^{1}$ P. Wasser, ${ }^{3}$ Y. Zhang, ${ }^{1}$ and S. Zoia ${ }^{1}$ \\ ${ }^{1}$ Max-Planck-Institut für Physik, Werner-Heisenberg-Institut, 80805 München, Germany \\ ${ }^{2}$ Physik-Institut, Universität Zürich, Wintherturerstrasse 190, CH-8057 Zürich, Switzerland \\ ${ }^{3}$ PRISMA Cluster of Excellence, Institute of Physics, Johannes Gutenberg University, D-55099 Mainz, Germany
}

(Received 28 December 2018; revised manuscript received 5 April 2019; published 25 July 2019)

\begin{abstract}
We evaluate analytically all previously unknown nonplanar master integrals for massless five-particle scattering at two loops, using the differential equations method. A canonical form of the differential equations is obtained by identifying integrals with constant leading singularities, in $D$ space-time dimensions. These integrals evaluate to $\mathbb{Q}$-linear combinations of multiple polylogarithms of uniform weight at each order in the expansion in the dimensional regularization parameter and are in agreement with previous conjectures for nonplanar pentagon functions. Our results provide the complete set of two-loop Feynman integrals for any massless $2 \rightarrow 3$ scattering process, thereby opening up a new level of precision collider phenomenology.
\end{abstract}

DOI: 10.1103/PhysRevLett.123.041603

Introduction.-The ever-improving experimental precision at the LHC challenges theoretical physicists to keep up with the accuracy of the corresponding theoretical predictions. In order for this to be possible, analytic expressions for higher-loop amplitudes play a crucial role. Among the processes that are investigated at hadron colliders, jet production observables offer unique opportunities for precision measurements. In particular, the ratio of threeand two-jet cross sections gives a measure of the strong coupling constant $\alpha_{S}\left(Q^{2}\right)$ at high energy scales $Q^{2}$ [1-6].

While many results for next-to-next-to leading order (NNLO) cross sections are available for $2 \rightarrow 2$ processes, higher multiplicity reactions are only beginning to be explored [7-15], so far mostly in the planar limit.

The situation was somewhat similar about 15 years ago at NLO, when novel theoretical ideas led to what is now called the "NLO revolution" [16]. Thanks to recent progress in quantum field theory methods, today we are at the brink of a NNLO revolution.

The new ideas include cutting-edge integral reduction techniques based on finite fields and algebraic geometry [17-19], a systematic mathematical understanding of special functions appearing in Feynman integrals [20,21], and their computation via differential equations [22] in the canonical form [23]. The latter in fact lead to simple iterated integral solutions that have uniform transcendental weight (UT), also called pure functions.

Published by the American Physical Society under the terms of the Creative Commons Attribution 4.0 International license. Further distribution of this work must maintain attribution to the author(s) and the published article's title, journal citation, and DOI. Funded by SCOAP ${ }^{3}$.
It is particularly interesting that many properties of the integrated functions can be anticipated from properties of the simpler Feynman loop integrands through the study of the so-called leading singularities [24]. A useful conjecture $[23,24]$ allows one to predict which Feynman integrals satisfy the canonical differential equation by analyzing their four-dimensional leading singularities. This can be done algorithmically [25].

It turns out that in complicated cases, especially when many scales are involved, the difference between treating the integrand as four or $D$ dimensional can become relevant. In particular, integrands whose numerators contain Gram determinants that vanish in four dimensions may spoil the UT property.

In this Letter we propose a new, refined criterion for finding the canonical form of the differential equations, and hence UT integrals. The method involves computing leading singularities in Baikov representation [26].

We apply our novel technique to the most complicated nonplanar massless five-particle integrals at NNLO. We explain how the UT basis is obtained and derive the canonical differential equation. We determine analytically the boundary values by requiring physical consistency. The solutions are found to be in agreement with a previous conjecture for nonplanar pentagon functions, and also with a previously conjectured second entry condition [27].

This result completes the analytic calculation of all master integrals required for three-jet production at hadron colliders to NNLO in QCD. We expect that our method will have many applications for multijet calculations in the near future.

Integral families.-Figure 1 shows the integral topologies needed for studying the scattering of five massless particles at two loops. The master integrals of the planar 


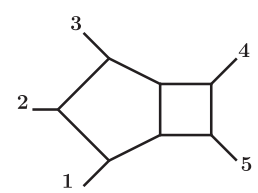

(a)

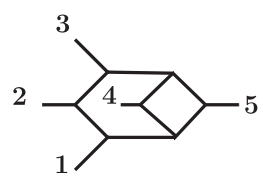

(b)

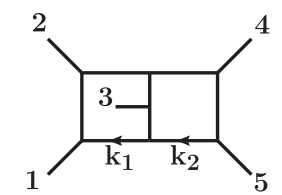

(c)

double pentagon pentabox

$$
\Omega=\sum_{I=\left(i_{1}, \ldots, i_{4 \ell}\right)} c_{I} d \log R_{i_{1}} \wedge \cdots \wedge d \log R_{i_{4 \ell}},
$$

where the $\mathbb{Q}$-valued constants $c_{I}$ are the leading singularities of $\Omega$.

In order to perform the loop integration in $D=4-2 \epsilon$ dimensions, where $\epsilon$ is the dimensional regulator, it is necessary to clarify how the integrand is to be defined away from four dimensions. For example, one may simply "upgrade" the loop momenta from four-dimensional to $D$-dimensional (abbreviated as 4D and DD) ones. We call this the "naive upgrade" of a 4D integrand. While this method is quite powerful in finding a UT basis, and indeed it has already found many successful applications $[23,39]$, the freedom involved in the upgrade can become important, especially for integrals with many kinematic scales. We first review the four-dimensional analysis, and then provide a method of fixing the freedom, while maintaining the advantages of the canonical differential equations method.

In this Letter, we use two techniques to find 4D $d \log$ integrals.

(1) The algorithm [25], which can decide if a given rational integrand can be cast in $d \log$ form Eq. (3). Starting from a generic ansatz for the numerator, this algorithm can classify all possible 4D $d \log$ integrals in a given family.

(2) Using computational algebraic geometry, we consider a generic ansatz for the numerator $N_{\text {even }}=\sum_{\alpha} c_{\alpha} m_{\alpha}$ of the parity-even or $N_{\text {odd }}=\sum_{\alpha} c_{\alpha} m_{\alpha} / \epsilon_{5}$ of the parity-odd $d \log$ integrals. Each $c_{\alpha}$ is a polynomial in $s_{i j}$, and $m_{\alpha}$ is a monomial in the scalar products. By requiring the $4 \mathrm{D}$ leading singularities of the ansatz to match a given list of rational numbers, we can use the module lift techniques [40] in computational algebraic geometry to calculate all $c_{\alpha}$ and to obtain a 4D $d \log$ basis. This method usually needs only a very simple ansatz, and the module lift can then be performed through the computer algebra system SINGULAR [41].

One interesting phenomenon is that, for the doublepentagon family, the naive upgrade of a 4D $d$ log integral is in general not UT. Let us take the 4D $d \log$ integrals presented in Ref. [42] as examples. The sum of the first and the fifth $d \log$ integral numerators for the double-pentagon diagram in Ref. [42], which we denote by $B_{1}+B_{5}$, does not yield a UT integral after the naive upgrade. This can be assessed from the explicit computation of the differential equation.

The obstruction of the naive upgrade implies that, in order to obtain UT integrals, we have to consider terms in the integrands which vanish as $D=4$. These terms can be conveniently constructed from Gram determinants involving the loop momenta $k_{1}$ and $k_{2}$ :

$G_{i j}=G\left(\begin{array}{c}k_{i}, p_{1}, p_{2}, p_{3}, p_{4} \\ k_{j}, p_{1}, p_{2}, p_{3}, p_{4}\end{array}\right), \quad$ with $\quad i, j \in\{1,2\}$. 
An integrand whose numerator is proportional to a combination of the different $G_{i j}$ explicitly vanishes in the $D \rightarrow 4$ limit. UT integral criteria based on 4D cuts or 4D $d \log$ constructions cannot detect these Gram determinants, and may yield inaccurate answers on whether an integral is UT in $D$ dimensions or not.

Instead, we develop a new $D$-dimensional criterion for UT integrals, based on the study of the cuts in Baikov representation. Our method analyzes the $D D$ leading singularities, and for a given 4D $d \log$ integral with 4D integrand $N /\left(D_{1} \ldots D_{k}\right)$, our criterion generates a $D \mathrm{D}$ integrand of the form

$$
\frac{\tilde{N}}{\tilde{D}_{1} \ldots \tilde{D}_{k}}+\frac{\tilde{S}}{\tilde{D}_{1} \ldots \tilde{D}_{k}},
$$

which is a UT integral candidate. Here the tilde sign denotes the naive upgrade, and $\tilde{S}$ is proportional to Gram determinants. We name Eq. (5) the refined upgrade of the 4D $d \log$ integrand $N /\left(D_{1} \ldots D_{k}\right)$. The details of this $D$-dimensional criterion based on Baikov cuts are given in the next section.

Applying our method to the top sector of the doublepentagon family leads to two observations.

(1) For any 4D double-pentagon $d \log$ in Ref. [42] we can find its refined upgrade from our DD UT criterion. We verified that such refined upgrades are indeed UT integrals by computing the differential equation. For example, the refined upgrade of $\left(B_{1}+B_{5}\right)$ is

$$
\begin{aligned}
& \left(\tilde{B}_{1}+\tilde{B}_{5}\right)+\frac{16 s_{45} G_{12}}{\epsilon_{5}^{2}} \\
& \quad \times\left(s_{12} s_{23}-s_{12} s_{15}+2 s_{12} s_{34}+s_{23} s_{34}+s_{15} s_{45}-s_{34} s_{45}\right) .
\end{aligned}
$$

(2) Some integrals with purely Gram-determinant numerators satisfy our DD UT criterion:

$\frac{s_{45}}{\epsilon_{5}}\left(G_{11}-G_{12}\right), \quad \frac{s_{12}}{\epsilon_{5}}\left(G_{22}-G_{12}\right), \quad \frac{s_{12}-s_{45}}{\epsilon_{5}} G_{12}$.

Once again we verified that these integrals are indeed UT by examining the differential equation.

Criterion for pure integrals from D-dimensional cuts.In this section we present our new criterion for UT integrals based on $D D$ cuts in the Baikov representation [26]. As we have already seen, this new criterion is sharper than the original 4D one, as it can also detect Gram determinants to which the latter is blind.

Let us recall that in the Baikov representation [26] the propagators of a $D D$ Feynman integrand are taken to be integration variables (Baikov variables). The $D \mathrm{D}$ leading singularities can thus be calculated easily by taking iterative residues. Then, our DD criterion for a UT integral is to require all the residues of its Baikov representation to be rational numbers.

For the double-pentagon integral family, the standard Baikov cut analysis [43,44], based on the two-loop Baikov representation, eventually leads to complicated threefold integrals. To avoid this computational difficulty, we adopt the loop-by-loop Baikov cut analysis [45].

For a double-pentagon integral with some numerator $N$, for instance, the integration can be separated loop by loop as

$I_{\mathrm{dp}}[N]=\int d^{D} k_{2} \frac{1}{D_{4} D_{5} D_{6}} \int d^{D} k_{1} \frac{N}{D_{1} D_{2} D_{3} D_{7} D_{8}}$.

The two-loop integral can thus be decomposed into a pentagon integral with loop momentum $k_{1}$ and external legs $p_{1}, p_{2}, p_{3}$, and $-k_{2}$, and a triangle integral with loop momentum $k_{2}$. Note that, if necessary, we might need to carry out a one-loop integral reduction for the numerator $N$ first, in order to make sure that the integrand contains no cross terms such as $k_{1} \cdot p_{4}$ or $k_{1} \cdot p_{5}$. As a consequence, $D_{9}$ drops out from the integrand.

We then apply the Baikov representation loop by loop; i.e., we change integration variables from the components of the loop momenta to 10 Baikov variables, $z_{i} \equiv D_{i}$, $i \in\{1, \ldots, 11\} \backslash\{9\}$. Once this is done, we can explore the $D D$ residues.

For instance, consider the double-pentagon integral $I_{\mathrm{dp}}\left[G_{12}\right]$. Its $4 \mathrm{D}$ leading singularities are all vanishing, and can therefore not determine whether $I_{\mathrm{dp}}\left[G_{12}\right]$ is UT or not. Conversely, by using our Baikov cut method, having integrated out the term $k_{1} \cdot p_{4}$, we get a Baikov integration with 10 variables. Taking the residues in $z_{i}=0, \forall i \in C$, where $C \subseteq\{1, \ldots, 8\}$, yields integrands which do not vanish in the $D \rightarrow 4$ limit. Using the algorithm [25], we systematically compute all possible residues of these integrands in the remaining variables, and make sure that there are no double poles. In this way we compute the leading singularities on different cuts, and find that they all evaluate to $\pm \epsilon_{5} /\left(s_{12}-s_{45}\right)$ or zero. As a result, we see that the integral

$$
\frac{s_{12}-s_{45}}{\epsilon_{5}} I_{\mathrm{dp}}\left[G_{12}\right]
$$

satisfies our DD criterion. We confirmed that Eq. (9) is indeed a UT integral by explicitly computing it from differential equations.

Similarly, we can use this loop-by-loop Baikov cut method to find the UT integral candidates listed in Eqs. (6) and (7), for which the 4D leading singularity calculation cannot give a definitive answer. All these candidates are subsequently proven to be UT by the differential equations.

It is worth noting that this DD Baikov cut analysis only involves basic integrand reduction and residue 
computations. We expect that this method, combined with the $d \log$ construction algorithm described in Ref. [25], will prove to be a highly efficient way of determining UT integral candidates for even more complicated diagrams in the future.

Master integrals and canonical differential equations.With the study of 4D $d \log$ integrals, and the novel $D \mathrm{D}$ Baikov cut analysis, we constructed a candidate UT integral basis for the double-pentagon family.

Through integration-by-parts identities, we find that the eight 4D $d \log \mathrm{s}$ in Ref. [42], after our refined upgrade, together with the three Gram-determinant integrals given in Eq. (7), span an eight-dimensional linear space. By combining the algorithm described in Ref. [25] and the computational algebraic geometry method, we easily find another linearly independent integral satisfying our $D D$ UT criterion. This completes the basis for the double-pentagon on the top sector. Subsector UT integrals are either found via Ref. [25] or taken from the literature $[9,29,30]$.

By differentiating our candidate UT basis for the doublepentagon family, we see that the differential equations are immediately in the canonical form [23]

$$
d \vec{I}\left(s_{i j} ; \epsilon\right)=\epsilon d \tilde{A}\left(s_{i j}\right) \vec{I}\left(s_{i j} ; \epsilon\right),
$$

without the need for any further basis change. This is the ultimate proof that our basis integrals are indeed UT.

We wish to emphasize here that the construction of the UT basis is done at the integrand level via Baikov cut analysis, and as such does not require the a priori knowledge of the differential equations.

It is also worth mentioning that the analytic inverse of the transformation matrix between our UT basis and the "traditional" basis from the Laporta algorithm was efficiently computed by means of the sparse linear algebra techniques described in Ref. [46].

Equation (10) can be further structured to the form

$$
d \vec{I}\left(s_{i j} ; \epsilon\right)=\epsilon\left(\sum_{k=1}^{31} a_{k} d \log W_{k}\left(s_{i j}\right)\right) \vec{I}\left(s_{i j} ; \epsilon\right),
$$

where $W_{k}$ are letters of the pentagon symbol alphabet conjectured in Ref. [27], and each $a_{k}$ is a $108 \times 108$ rational number matrix.

We consider the integrals in the $s_{12}$ scattering region. The latter is defined by positive $s$-channel energies, $\left\{s_{12}, s_{34}, s_{45}, s_{35}\right\} \geq 0$, and negative $t$-channel energies, $\left\{s_{23}, s_{24}, s_{25}, s_{13}, s_{14}, s_{15}\right\} \leq 0$, as well as reality of particle momenta, which translates to $\Delta \leq 0$.

We choose a boundary point

$$
X_{0}=\{3,-1,1,1,-1\}
$$

inside this region. We determine the boundary values of the integrals by requiring physical consistency, as described in
Ref. [30]. This yields a system of equations for the boundary constants at $X_{0}$, whose coefficients are Goncharov polylogarithms. We evaluate the latter to high precision using GINAC [47]. The values at $X_{0}$ were validated successfully with the help of SECDEC [48].

The full result for the integrals is again written in terms of Goncharov polylogarithms. For reference, we provide numerical values for all integrals at the symmetric point $X_{0}$, as well as for an asymmetric point,

$$
X_{1}=\left\{4,-\frac{113}{47}, \frac{281}{149}, \frac{349}{257},-\frac{863}{541}\right\} .
$$

The values, given in Supplemental Material [49], have at least 50 digit precision. Here we display the results for integral $I_{107}$ :

$I_{107}\left(X_{0}, \epsilon\right)=16.383606637078885171 i+\mathcal{O}(\epsilon)$,

$I_{107}\left(X_{1}, \epsilon\right)=6.9362922441923047974 i+\mathcal{O}(\epsilon)$.

From their leading order term in $\epsilon$ of the boundary values, one can immediately write down the symbol of the integrals. This has also been computed independently in Ref. [50], and has already been employed in the computation of two-loop five-point amplitudes in $\mathcal{N}=4$ superYang-Mills theory [50,51] and $\mathcal{N}=8$ supergravity [52,53] at symbol level. We observe that the second entry condition conjectured in Ref. [27] is indeed satisfied.

We provide the UT basis for the double-pentagon family, the $\tilde{\mathcal{A}}$ matrix of the canonical differential Eq. (10), and the boundary values at $X_{0}$ and $X_{1}$ in Supplemental Material [49].

Discussion and outlook.-In this Letter, we computed analytically the master integrals of the last missing integral family needed for massless five-particle scattering amplitudes at two loops. We applied the canonical differential equation method [23], supplemented with a novel strategy for finding integrals evaluating to pure functions based on the analysis of $D \mathrm{D}$ leading singularities in Baikov representation.

Our calculation confirms the previously conjectured pentagon functions alphabet and second entry condition [27]. Our result implies the latter is a property of individual Feynman integrals, not only of full amplitudes. It will be interesting to find a field theory explanation of this condition, perhaps along the lines of the Steinmann relations.

With our result, all master integrals relevant for three-jet production at NNLO are now known analytically. Moreover, they are ready for numerical evaluation in physical scattering regions. This opens the door to computing full $2 \rightarrow 3$ scattering amplitudes at two loops.

We expect that our $D D$ Baikov cut analysis will prove to be a powerful method to find Feynman integrals evaluating 
to pure functions, in particular, for integral families involving many scales. We expect it will have many further applications for multiparticle amplitudes, e.g., for $H+2 j$ and $V+2 j$ productions, and other multiscale processes relevant for collider physics.

We are indebted to Gudrun Heinrich and Stephan Jahn for providing numerical checks and for help with using SECDEC. Y.Z. thanks Alessandro Georgoudis and Yingxuan $\mathrm{Xu}$ for enlightening discussions. This research received funding from the Swiss National Science Foundation (Ambizione Grant No. PZ00P2 161341), the European Research Council (ERC) under the European Union's Horizon 2020 Research, and Innovation Programme (Grant Agreement No. 725110), "Novel Structures in Scattering Amplitudes." J.M. H., Y.Z., and S.Z. also wish to thank the Galileo Galilei Institute for hospitality during the workshop "Amplitudes in the LHC Era."

[1] J. R. Andersen et al., in Physics at TeV Colliders Standard Model Working Group Report, Proceedings of the 10th Les Houches Workshop on Physics at TeV Colliders (PhysTeV 2017), Les Houches, France, 2017(2018), https://inspirehep .net/record/1663483.

[2] G. Aad et al. (ATLAS Collaboration), Phys. Lett. B 750, 427 (2015).

[3] M. Aaboud et al. (ATLAS Collaboration), Eur. Phys. J. C 77, 872 (2017).

[4] M. Aaboud et al. (ATLAS Collaboration), Phys. Rev. D 98, 092004 (2018).

[5] V. Khachatryan et al. (CMS Collaboration), Eur. Phys. J. C 75, 186 (2015).

[6] S. Chatrchyan et al. (CMS Collaboration), Eur. Phys. J. C 73, 2604 (2013).

[7] S. Badger, H. Frellesvig, and Y. Zhang, J. High Energy Phys. 12 (2013) 045.

[8] S. Badger, G. Mogull, A. Ochirov, and D. O'Connell, J. High Energy Phys. 10 (2015) 064.

[9] T. Gehrmann, J. M. Henn, and N. A. Lo Presti, Phys. Rev. Lett. 116, 062001 (2016); 116, 189903(E) (2016).

[10] S. Badger, C. Brønnum-Hansen, H. B. Hartanto, and T. Peraro, Phys. Rev. Lett. 120, 092001 (2018).

[11] S. Abreu, F. Febres Cordero, H. Ita, B. Page, and M. Zeng, Phys. Rev. D 97, 116014 (2018).

[12] R. H. Boels, Q. Jin, and H. Luo, arXiv:1802.06761.

[13] S. Abreu, F. Febres Cordero, H. Ita, B. Page, and V. Sotnikov, J. High Energy Phys. 11 (2018) 116.

[14] S. Badger, C. Brønnum-Hansen, H. B. Hartanto, and T. Peraro, J. High Energy Phys. 01 (2019) 186.

[15] S. Abreu, J. Dormans, F. F. Cordero, H. Ita, and B. Page, Phys. Rev. Lett. 122, 082002 (2019).

[16] R. K. Ellis, Z. Kunszt, K. Melnikov, and G. Zanderighi, Phys. Rep. 518, 141 (2012).

[17] A. von Manteuffel and R. M. Schabinger, Phys. Lett. B 744, 101 (2015).

[18] T. Peraro, J. High Energy Phys. 12 (2016) 030.

[19] Y. Zhang, J. High Energy Phys. 09 (2012) 042.
[20] A. B. Goncharov, M. Spradlin, C. Vergu, and A. Volovich, Phys. Rev. Lett. 105, 151605 (2010).

[21] C. Duhr, H. Gangl, and J. R. Rhodes, J. High Energy Phys. 10 (2012) 075.

[22] T. Gehrmann and E. Remiddi, Nucl. Phys. B580, 485 (2000).

[23] J. M. Henn, Phys. Rev. Lett. 110, 251601 (2013).

[24] N. Arkani-Hamed, J. L. Bourjaily, F. Cachazo, and J. Trnka, J. High Energy Phys. 06 (2012) 125.

[25] P. Wasser, M.Sc. thesis, Johannes Gutenberg University Mainz, 2016, https://publications.ub.uni-mainz.de/theses/ frontdoor.php?source opus $=100001967$.

[26] P. A. Baikov, Phys. Lett. B 385, 404 (1996).

[27] D. Chicherin, J. Henn, and V. Mitev, J. High Energy Phys. 05 (2018) 164.

[28] C. G. Papadopoulos, D. Tommasini, and C. Wever, J. High Energy Phys. 04 (2016) 078.

[29] T. Gehrmann, J. M. Henn, and N. A. Lo Presti, J. High Energy Phys. 10 (2018) 103.

[30] D. Chicherin, T. Gehrmann, J. M. Henn, N. A. Lo Presti, V. Mitev, and P. Wasser, J. High Energy Phys. 03 (2019) 042.

[31] D. Chicherin, J. M. Henn, and E. Sokatchev, Phys. Rev. Lett. 121, 021602 (2018).

[32] D. Chicherin, J. M. Henn, and E. Sokatchev, J. High Energy Phys. 09 (2018) 012.

[33] S. Abreu, B. Page, and M. Zeng, J. High Energy Phys. 01 (2019) 006.

[34] A. V. Smirnov, J. High Energy Phys. 10 (2008) 107.

[35] A. von Manteuffel and C. Studerus, arXiv:1201.4330.

[36] A. Georgoudis, K. J. Larsen, and Y. Zhang, Comput. Phys. Commun. 221, 203 (2017).

[37] T. Gehrmann and E. Remiddi, Nucl. Phys. B601, 248 (2001).

[38] T. Gehrmann and E. Remiddi, Nucl. Phys. B601, 287 (2001).

[39] J. M. Henn, J. Phys. A 48, 153001 (2015).

[40] G.-M. Greuel and G. Pfister, A Singular Introduction to Commutative Algebra, 2nd ed. (Springer Publishing Company, Incorporated, 2007).

[41] W. Decker, G.-M. Greuel, G. Pfister, and H. Schönemann, SINGULAR 4-1-1-A computer algebra system for polynomial computations, http://www.singular.uni-kl.de.

[42] Z. Bern, E. Herrmann, S. Litsey, J. Stankowicz, and J. Trnka, J. High Energy Phys. 06 (2016) 098.

[43] J. Bosma, M. Sogaard, and Y. Zhang, J. High Energy Phys. 08 (2017) 051.

[44] M. Harley, F. Moriello, and R. M. Schabinger, J. High Energy Phys. 06 (2017) 049.

[45] H. Frellesvig and C. G. Papadopoulos, J. High Energy Phys. 04 (2017) 083.

[46] J. Boehm, A. Georgoudis, K. J. Larsen, H. Schönemann, and Y. Zhang, J. High Energy Phys. 09 (2018) 024.

[47] C. W. Bauer, A. Frink, and R. Kreckel, J. Symb. Comput. 33, 1 (2002).

[48] S. Borowka, G. Heinrich, S. P. Jones, M. Kerner, J. Schlenk, and T. Zirke, Comput. Phys. Commun. 196, 470 (2015).

[49] See Supplemental Material at http://link.aps.org/ supplemental/10.1103/PhysRevLett.123.041603 for the 
definition of the master integrals and of the $\tilde{A}$ matrix fulfilling the canonical differential equations in Eq. (10), and for the values of the master integrals at the points defined in Eqs. (12) and (13).

[50] S. Abreu, L. J. Dixon, E. Herrmann, B. Page, and M. Zeng, Phys. Rev. Lett. 122, 121603 (2019).
[51] D. Chicherin, T. Gehrmann, J. M. Henn, P. Wasser, Y. Zhang, and S. Zoia, Phys. Rev. Lett. 122, 121602 (2019).

[52] D. Chicherin, T. Gehrmann, J. M. Henn, P. Wasser, Y. Zhang, and S. Zoia, J. High Energy Phys. 03 (2019) 115.

[53] S. Abreu, L. J. Dixon, E. Herrmann, B. Page, and M. Zeng, J. High Energy Phys. 03 (2019) 123. 